\title{
Spinning up black holes with super-critical accretion flows
}

\author{
A. Sądowski ${ }^{1}$, M. Bursa ${ }^{2}$, M. Abramowicz ${ }^{1,2,3,4}$, W. Kluźniak ${ }^{1}$, J.-P. Lasota ${ }^{5,6}$, R. Moderski ${ }^{1}$, and M. Safarzadeh ${ }^{3,7}$ \\ 1 Nicolaus Copernicus Astronomical Center, Polish Academy of Sciences, Bartycka 18, 00-716 Warszawa, Poland \\ e-mail: [as; wlodek; moderski]@camk.edu.pl \\ 2 Astronomical Institute, Academy of Sciences of the Czech Republic, Boční II 1401/1a, 141-31 Praha 4, Czech Republic \\ e-mail: bursa@astro.cas.cz \\ 3 Department of Physics, Göteborg University, 412-96 Göteborg, Sweden \\ e-mail: marek.abramowicz@physics.gu.se \\ ${ }^{4}$ Department of Physics, Silesian University at Opava, Bezručovo náměstí 1150/13, 74601 Opava, Czech Republic \\ 5 Institut d'Astrophysique de Paris, UMR 7095 CNRS, UPMC Univ. Paris 06, 98bis Bd Arago, 75014 Paris, France \\ e-mail: lasota@iap.fr \\ ${ }^{6}$ Jagiellonian University Observatory, ul. Orla 171, 30-244 Kraków, Poland \\ 7 Department of Physics and Astronomy, Johns Hopkins University, 3400 N. Charles Street Baltimore, MD 21218, USA \\ e-mail: mts@pha.jhu.edu
}

Received 11 February 2011 / Accepted 24 May 2011

\section{ABSTRACT}

\begin{abstract}
We study the process of spinning up black holes by accretion from slim disks for a wide range of accretion rates. We show that for super-Eddington accretion rates and low values of the viscosity parameter $\alpha(\lesssim 0.01)$ the limiting value of the dimensionless spin parameter $a_{*}$ can reach values higher than $a_{*}=0.9978$ inferred by Thorne in his seminal study. For $\dot{M}=10 \dot{M}_{\text {Edd }}$ and $\alpha=0.01$, spin equilibrium is reached at $a_{*}=0.9994$. We show that the equilibrium spin value depends strongly on the assumed value of $\alpha$. We also prove that for high accretion rates the impact of captured radiation on spin evolution is negligible.
\end{abstract}

Key words. black hole physics - accretion, accretion disks

\section{Introduction}

Astrophysical black holes (BHs) are very simple objects - they can be described by just two parameters: mass $M$ and angular momentum $J$ (usually described by the dimensionless spin parameter $a_{*} \equiv a / M=J / M^{2}$ ). In isolation, BHs conserve the birth values of these parameters but are often surrounded by accretion disks and experience both mass and angular momentum change, e.g., in close binaries or in active galactic nuclei. Accretion of matter always increases the BH's irreducible mass and may change its angular momentum. The sign of this change and its value depend on the (relative) sign of accreted angular momentum and the balance between the accretion of matter and various processes extracting the BH's rotational energy and angular momentum.

The question about the maximal possible spin of an object represented by the Kerr solution of the Einstein equation is of fundamental and practical (observational) interest. First, a spin $a_{*}>1$ corresponds to a naked singularity and not to a black hole. According to the Penrose cosmic censorship conjecture, naked singularities cannot form through actual physical processes, i.e. singularities in the Universe (except for the initial one in the Big Bang) are always surrounded by event horizons (Wald 1984). This hypothesis has yet to be proven.

In any case, the "third law" of BH thermodynamics (Bardeen et al. 1973) asserts that a BH cannot be spun-up in a finite time to the extreme spin value $a_{*}=1$. Determining the maximum value of BH spin is also of practical interest because the radiative efficiency of disk accretion depends on the BH's spin value. For example, for the "canonical" value $a_{*}=0.9978$ (see below) it is about $\eta \approx 32 \%$, while for $a_{*} \rightarrow 1$ one has $\eta \rightarrow 42 \%$. Bañados et al. (2009) showed that the energy of the center-of-mass collision of two particles colliding arbitrary close to the BH horizon, grows to infinity ${ }^{1}$ when $a_{*} \rightarrow 1$.

A definitive study of the BH spin evolution will only be possible when reliable, non-stationary models of accretion disks and jet emission mechanisms are established. For now, one has to use simplified analytical or numerical models.

Thorne (1974) used the model of a radiatively efficient, geometrically thin accretion disk (Novikov \& Thorne 1973) to evaluate $\mathrm{BH}$ spin evolution taking into account the decelerating impact of disk-emitted photons. The maximum value obtained to date $a_{*}=0.9978$, has been regarded as the canonical value for the maximal BH spin. In this work, we generalize Thorne's approach, using hydrodynamical models of advective, $\alpha$-viscosity, optically thick accretion disks ("slim disks") to calculate maximum BH spin values for a large range of accretion rates. Following Thorne (1974), we assume that accretion of matter and radiation captured by the $\mathrm{BH}$ are the only mechanisms affecting its rotation. Thus, we neglect any impact of large-scale magnetic fields (a discussion of the applicability of slim disks is presented in Sect. 6). We show that for sufficiently high accretion rates the limiting $\mathrm{BH}$ spin differs from the canonical value.

We begin with a short discussion of previous work devoted to the evolution of BH spin. In Sect. 2, we present formulae

\footnotetext{
${ }^{1}$ Of more fundamental interest is that the proper geodesic distances $D$ between the marginally stable orbit (the innermost stable orbit, ISCO) and several other special Keplerian orbits relevant to accretion disk structure tend to infinity $D \rightarrow \infty$ when $a_{*} \rightarrow 1$ (Bardeen et al. 1972).
} 
for a general tetrad (an orthonormal set of four vector fields, one timelike and three spacelike) of an observer comoving with the accreting gas along the arbitrary photosphere surface. In Sect. 3, we give basic equations describing the BH spin evolution. Section 4 describes the model of slim accretion disks. In Sect. 5, we present and discuss the terminal spin values for all the models considered. Finally, in Sect. 6 we summarize our results.

\subsection{Previous studies}

A number of authors have studied the BH spin evolution resulting from disk accretion. Bardeen (1970) initiated this field of research by solving equations describing the $\mathrm{BH}$ spin evolution for accretion from the marginally stable orbit. Neglecting the effects of radiation, he proved that this accretion could spin-up the $\mathrm{BH}$ arbitrarily close to $a_{*}=1$. Once the classical models of accretion disks were formulated (Shakura \& Sunyaev 1973; Novikov $\&$ Thorne 1973), it was possible to account properly for the decelerating impact of radiation (frame dragging makes counterrotating photons more likely to be captured by the $\mathrm{BH}$ ). As mentioned above, Thorne (1974) performed this study and obtained for an isotropically emitting thin disk the terminal $\mathrm{BH}$ spin $a_{*}=0.9978$, independently of the accretion rate. The original study by Thorne was followed by many papers, some of which are briefly mentioned below.

The first to challenge the universality of Thorne's limit were Abramowicz \& Lasota (1980), who showed that geometrically thick accretion disks may spin up BHs to terminal spin values much closer to unity than the canonical $a_{*}=0.9978$. Their simple argument was based on models by Kozlowski et al. (1978), who showed that for high accretion rates the inner edge of a disk may be located inside the marginally stable orbit, and in fact, with increasing accretion rate, arbitrarily close to the marginally bound orbit. However, this conclusion assumed implicitly a low viscosity parameter $\alpha$, whereas for high viscosities the situation is more complicated (see Abramowicz et al. 2010, and references therein).

Moderski et al. (1998) assessed the impact of possible interaction between the disk magnetic field and the BH through the Blandford-Znajek process. They showed that the terminal spin value may be decreased to any, arbitrarily small value, if the disk magnetic field is strong enough. Given the current lack of selfconsistent and reliable models of accretion disks with large-scale magnetic fields, a more detailed study cannot be performed. The situation may be further complicated by energy extraction from the inner parts of accretion disks and the magnetic transport of angular momentum (see Livio et al. 1999; Ghosh \& Abramowicz 1997; and compare with McKinney \& Narayan 2007).

Popham \& Gammie (1998) studied the spinning-up of BHs by optically thin advection dominated accretion flows (ADAFs). They neglected the contribution of radiation to $\mathrm{BH}$ spin because such accretion disks are radiatively inefficient. They found that the terminal value of $\mathrm{BH}$ spin is very sensitive to the assumed value of the viscosity parameter $\alpha$ and may vary between 0.8 and 1.0. Gammie et al. (2004), in addition to comprehensively summarizing the different ways of spinning up supermassive BHs, presented results based on a set of relativistic magnetohydrodynamical (GRMHD) simulations (with no radiation included) obtaining a terminal spin of $a_{*}=0.93$.

The cosmological evolution of the spins of supermassive BHs caused by hierarchical mergers and thin-disk accretion episodes has been intensively studied. Although Volonteri et al. (2005) arrived at the conclusion that accretion tends to spin-up BHs close to $a_{*}=1$, as opposed to mergers, which, on the average, do not influence the spin, subsequent studies by, e.g., Volonteri et al. (2007), King et al. (2008) and Berti \& Volonteri (2008) showed that the situation is more complex, the final spin values depending on the details of the history of the accretion events (see also Fanidakis et al. 2011).

Belczynski et al. (2008) applied population synthesis methods to estimate $\mathrm{BH}$ spins in coalescing compact star binaries. Basing their calculations on results of radiation-hydrodynamic simulations of thick accretion disks by Ohsuga (2007), they neglected the impact of radiation on $\mathrm{BH}$ spin and assumed that gas is transferred from the innermost stable orbit conserving Keplerian angular momentum. They showed that the spin parameter $a_{*}$ resulting from the coalescence is not expected to exceed 0.5 for those BHs that are not spun-up during the star collapse.

Li et al. (2005) included the radiation returning to the disk in the thin-disk model of Novikov \& Thorne and calculated the spin-up limit for the $\mathrm{BH}$ assuming radiation crossing the equatorial plane inside the marginally stable orbit is advected onto the BH. Their result ( $\left.a_{*}=0.9983\right)$ differs slightly from Thorne's result, thus showing that returning radiation has only a slight impact on the process of spinning-up BHs. In our study, we use advective, optically thick solutions of accretion disks and account for photons captured by the $\mathrm{BH}$ in detail. However, we neglect the impact on the disk structure of the returning radiation.

\section{The tetrad}

We base this work on slim accretion disks, which are not razorthin and have an angular momentum profile that is not Keplerian (for details about the assumptions made and the disk appearance see Sect. 4). Therefore, photons are not emitted from matter in Keplerian orbits in the equatorial plane and the classical expressions for photon momenta (e.g., Misner et al. 1973) cannot be applied. To properly describe the momentum components of emitted photons, we need a tetrad for the comoving observer instantaneously located at the disk photosphere. Below we give the explicit expression for the components of such a tetrad assuming time and axis symmetries. A detailed derivation is given in Appendix A.

We choose the following comoving tetrad

$e_{(\alpha)}^{i}=\left[u^{i}, N^{i}, \kappa^{i}, S^{i}\right]$

where $u^{i}$ is the four-velocity of matter, $N^{i}$ is a unit vector in the $[r, \theta]$ plane that is orthogonal to the photosphere, $\kappa^{i}$ is a unit vector in the $[t, \phi]$ plane that is orthogonal to $u^{i}$, and $S^{i}$ is a unit vector orthogonal to $u^{i}, N^{i}$, and $\kappa^{i}$.

The tetrad components are given by

$$
\begin{aligned}
N^{r} & =\frac{\mathrm{d} \theta_{*}}{\mathrm{~d} r}\left(-g_{\theta \theta}\right)^{-1 / 2}\left[1+\frac{g_{r r}}{g_{\theta \theta}}\left(\frac{\mathrm{d} \theta_{*}}{\mathrm{~d} r}\right)^{2}\right]^{-1 / 2}, \\
N^{\theta} & =\left(-g_{\theta \theta}\right)^{-1 / 2}\left[1+\frac{g_{r r}}{g_{\theta \theta}}\left(\frac{\mathrm{d} \theta_{*}}{\mathrm{~d} r}\right)^{2}\right]^{-1 / 2}, \\
u^{i} & =\frac{\eta^{i}+\Omega \xi^{i}+v S_{*}^{i}}{\sqrt{g_{t t}+\Omega g_{\phi \phi}(\Omega-2 \omega)-v^{2}}} \\
\kappa^{i} & =\frac{\left(l \eta^{i}+\xi^{i}\right)}{\left[-g_{\phi \phi}(1-\Omega l)(1-\omega l)\right]^{1 / 2}} \\
S^{i} & =\left(1+\tilde{A}^{2} v^{2}\right)^{-1 / 2}\left(\tilde{A} v u^{i}+S_{*}^{i}\right)
\end{aligned}
$$


where $\theta=\theta_{*}(r)$ defines the location of the photosphere, $\eta_{i}$ and $\xi_{i}$ are the Killing vectors, $l=u_{\phi} / u_{t}, \Omega=u^{\phi} / u^{t}, \omega$ is the frequency of frame-dragging, and the expressions for $v$ and $S_{*}^{i}$ are given in Eqs. (A.10) and (A.4), respectively.

\section{Spin evolution}

\subsection{Basic equations}

The equations describing the evolution of dimensionless $\mathrm{BH}$ spin $a_{*}$ with respect to the $\mathrm{BH}$ energy $M$ and the accreted rest-mass $M_{0}$ are (Thorne 1974)

$$
\begin{aligned}
\frac{\mathrm{d} a_{*}}{\mathrm{~d} \ln M} & =\frac{\mathrm{d} J / M^{2}}{\mathrm{~d} \ln M}=\frac{1}{M} \frac{\dot{M}_{0} u_{\phi}+\left(\frac{\mathrm{d} J}{\mathrm{~d} t}\right)_{\mathrm{rad}}}{\dot{M}_{0} u_{t}+\left(\frac{\mathrm{d} M}{\mathrm{~d} t}\right)_{\mathrm{rad}}}-2 a_{*}, \\
\frac{\mathrm{d} M}{\mathrm{~d} M_{0}} & =u_{t}+\left(\frac{\mathrm{d} M}{\mathrm{~d} t}\right)_{\mathrm{rad}} / \dot{M}_{0} .
\end{aligned}
$$

The energy and angular momentum of $\mathrm{BH}$ increase due to the capture of photons according to the formulae

$$
\begin{aligned}
(\mathrm{d} M)_{\mathrm{rad}} & =\int_{\text {disk }} T^{i k} \eta_{k} N_{i} \mathrm{~d} S, \\
(\mathrm{~d} J)_{\mathrm{rad}} & =\int_{\text {disk }} T^{i k} \xi_{k} N_{i} \mathrm{~d} S,
\end{aligned}
$$

where $\eta_{k}$ and $\xi_{k}$ are the Killing vectors connected with time and axial symmetries, respectively, $T^{i k}$ is the stress-energy tensor of photons, which is taken to be non-zero only for photons crossing the $\mathrm{BH}$ horizon, and $\mathrm{d} S$ is the "volume element" in the hypersurface orthogonal to $N^{i}$, which is given by Eq. (B.8).

From Eqs. (9) and (10), it follows that

$$
\begin{aligned}
\left(\frac{\mathrm{d} M}{\mathrm{~d} t}\right)_{\mathrm{rad}} & =\int_{0}^{2 \pi} \int_{r_{\text {in }}}^{r_{\text {out }}} T^{i k} \eta_{k} N_{i} \mathrm{~d} \tilde{S}, \\
\left(\frac{\mathrm{d} J}{\mathrm{~d} t}\right)_{\mathrm{rad}} & =\int_{0}^{2 \pi} \int_{r_{\text {in }}}^{r_{\text {out }}} T^{i k} \xi_{k} N_{i} \mathrm{~d} \tilde{S},
\end{aligned}
$$

where

$$
\mathrm{d} \tilde{\mathrm{S}}=\mathrm{d} \phi \mathrm{d} r\left(g_{t \phi}^{2}-g_{t t} g_{\phi \phi}\right)^{1 / 2} \sqrt{g_{r r}+g_{\theta \theta}\left(\frac{\mathrm{d} \theta_{*}}{\mathrm{~d} r}\right)^{2}} .
$$

\subsection{Stress energy tensor in the comoving frame}

We select the tetrad given in Eq. (1)

$$
\begin{array}{ll}
e_{(0)}^{i}=u^{i} & e_{(1)}^{i}=N^{i} \\
e_{(2)}^{i}=\kappa^{i} & e_{(3)}^{i}=S^{i} .
\end{array}
$$

The disk properties, e.g., the emitted flux, are usually given in the comoving frame defined by Eq. (14). The stress tensor components in the two frames (Boyer-Lindquist and comoving) are related in the following way:

$$
T^{i k}=T^{(\alpha)(\beta)} e_{(\alpha)}^{i} e_{(\beta)}^{k} .
$$

The stress tensor in the comoving frame is

$T^{(\alpha)(\beta)}=2 \int_{0}^{\pi / 2} \int_{0}^{2 \pi} I_{0} S C \pi^{(\alpha)} \pi^{(\beta)} \sin \tilde{a} \mathrm{~d} \tilde{a} \mathrm{~d} \tilde{b}$, where $I_{0} S=I_{0}(r) S(\tilde{a}, \tilde{b})$ is the intensity of the emitted radiation, $\tilde{a}$ and $\tilde{b}$ are the angles between the emission vector and the $N^{i}$ and $S^{i}$ vectors, respectively, $C$ is the capture function defined in Sect. 3.4, the factor 2 occurs because the disk emission comes from both sides of the disk, and $\pi^{(\alpha)}=p^{(\alpha)} / p^{(0)}$ are the normalized components of the photon four-momentum in the comoving frame. The last set of parameters are given by the relations (Thorne 1974)

$\pi^{(0)}=1$,

$\pi^{(1)}=\cos \tilde{a}$,

$\pi^{(2)}=\sin \tilde{a} \cos \tilde{b}$,

$\pi^{(3)}=\sin \tilde{a} \sin \tilde{b}$.

Equations (11) and (12) take the form

$$
\begin{aligned}
\left(\frac{\mathrm{d} M}{\mathrm{~d} t}\right)_{\mathrm{rad}} & =\int_{\mathrm{disk}} T^{(\alpha)(\beta)} e_{(\alpha)}^{i} e_{(\beta)}^{k} \eta_{k} N_{i} \mathrm{~d} \tilde{S}, \\
\left(\frac{\mathrm{d} J}{\mathrm{~d} t}\right)_{\mathrm{rad}} & =\int_{\mathrm{disk}} T^{(\alpha)(\beta)} e_{(\alpha)}^{i} e_{(\beta)}^{k} \xi_{k} N_{i} \mathrm{~d} \tilde{S},
\end{aligned}
$$

where $e_{(\alpha)}^{i}$ is our local frame tetrad given by Eq. (14).

Taking into account the relations

$$
\begin{aligned}
\pi^{(\alpha)} & =\pi^{j} e_{j}^{(\alpha)}, \\
e_{j}^{(\alpha)} e_{(\alpha)}^{i} & =\delta_{j}^{i},
\end{aligned}
$$

we have

$$
\begin{aligned}
& \pi^{(\alpha)} e_{(\alpha)}^{i} N_{i}=\pi^{(\alpha)} \delta_{(\alpha)}^{(1)}=\pi^{(1)}=\cos \tilde{a}, \\
& \pi^{(\beta)} e_{(\beta)}^{k} \eta_{k}=\pi^{j} e_{j}^{(\beta)} e_{(\beta)}^{k} \eta_{k}=\pi^{j} \delta_{j}^{k} \eta_{k}=\pi^{k} \eta_{k}=\pi_{t}, \\
& \pi^{(\beta)} e_{(\beta)}^{k} \xi_{k}=\pi^{j} e_{j}^{(\beta)} e_{(\beta)}^{k} \xi_{k}=\pi^{j} \delta_{j}^{k} \xi_{k}=\pi^{k} \xi_{k}=\pi_{\phi},
\end{aligned}
$$

where,

$\pi_{t}=\pi^{(i)} e_{(i)}^{t} g_{t t}+\pi^{(i)} e_{(i)}^{\phi} g_{t \phi}$,
$\pi_{\phi}=\pi^{(i)} e_{(i)}^{t} g_{t \phi}+\pi^{(i)} e_{(i)}^{\phi} g_{\phi \phi}$.

Therefore, Eqs. (18) and (19) may be finally expressed as

$$
\begin{aligned}
\left(\frac{\mathrm{d} M}{\mathrm{~d} t}\right)_{\mathrm{rad}}= & 4 \pi \int_{r_{\text {in }}}^{r_{\text {out }}} \int_{0}^{2 \pi} \int_{0}^{\pi / 2} I_{0} S C \pi_{t} \\
& \times \cos \tilde{a} \sin \tilde{a} \mathrm{~d} \tilde{a} \mathrm{~d} \tilde{b} \sqrt{\tilde{g}} \mathrm{~d} r \\
\left(\frac{\mathrm{d} J}{\mathrm{~d} t}\right)_{\mathrm{rad}}= & 4 \pi \int_{r_{\text {in }}}^{r_{\text {out }}} \int_{0}^{2 \pi} \int_{0}^{\pi / 2} I_{0} S C \pi_{\phi} \\
& \times \cos \tilde{a} \sin \tilde{a} \mathrm{~d} \tilde{a} \mathrm{~d} \tilde{b} \sqrt{\tilde{g}} \mathrm{~d} r
\end{aligned}
$$

where

$$
\sqrt{\tilde{g}} \equiv\left(g_{t \phi}^{2}-g_{t t} g_{\phi \phi}\right)^{1 / 2} \sqrt{g_{r r}+g_{\theta \theta}\left(\frac{\mathrm{d} \theta_{*}}{\mathrm{~d} r}\right)^{2}} .
$$




\subsection{Emission}

The intensity of local radiation may be identified with the flux emerging from the disk surface

$I_{0}=F(r)$.

The angular emission factor $S$ was taken by Thorne (1974) to be

$S(\tilde{a}, \tilde{b})= \begin{cases}1 / \pi & \text { isotropic } \\ (3 / 7 \pi)(1+2 \cos \tilde{a}) & \text { limb darkening }\end{cases}$

for isotropic and limb-darkened cases, respectively. In this work, we assume that the radiation is emitted isotropically.

\subsection{Capture function}

The BH energy and angular momentum are affected only by photons crossing the BH horizon. Following Thorne (1974), we define the capture function $C$

$C=\left\{\begin{array}{l}1 \text { if the photon hits the } \mathrm{BH}, \\ 0 \text { in the opposite case. }\end{array}\right.$

Herein, we calculate $C$ in two ways. First, we use the original Thorne (1974) algorithm modified to account for emission out of the equatorial plane. For this purpose, we calculate the constants of motion, $j$ and $k$, for a geodesic orbit of a photon using

$j=a_{*}^{2}+a_{*}\left(\pi_{\phi} / M \pi_{t}\right)$,

$k=\frac{1}{\left(M \pi_{t}\right)^{2}}\left[\pi_{\theta}^{2}-\left(\pi_{\phi}+a_{*} M \pi_{t} \sin \theta_{*}\right)^{2} / \sin ^{2} \theta_{*}\right]$

which replaces Thorne's Eq. (A10). This approach does not take into account the effects of returning radiation, i.e., a photon hitting the disk surface is assumed to continue its motion. This treatment is inappropriate for optically thick disks - returning photons are most likely absorbed or advected towards the $\mathrm{BH}$.

To assess the importance of this inconsistency, we adopt two additional algorithms for calculating $C$. Using photon equations of motion, we determine whether the photon hits the disk surface (Bursa 2006). We then make one of two assumptions, either the angular momentum and energy of all "returning" photons are advected onto the $\mathrm{BH}\left(C_{1}\right)$, or all are re-emitted carrying away their original angular momentum and energy, and never hit the $\mathrm{BH}\left(C_{2}\right)$. In this way, we establish two limiting cases allowing us to assess the impact of the returning radiation.

We note that for a fully consistent treatment of the returning radiation (as in $\mathrm{Li}$ et al. 2005, for geometrically thin disks) it is not enough to modify the capture function, but that finding a solution for the whole structure of a self-irradiated accretion disk is instead necessary. The latter has not yet been done for luminous and geometrically thick disks. We are currently working on implementing such a scheme and will study its impact on BH spin evolution in an upcoming paper.

\section{Slim accretion disks}

\subsection{Equations}

We now introduce slim disk equations. They were derived originally by Lasota (1994) and improved e.g., by Abramowicz et al. (1996) and Gammie \& Popham (1998). Here, we follow Kato et al. (2008) and assume the polytropic equation of state when performing vertical integration. The formalism we use here was adopted from Sạdowski et al. (2011).

In the structure equations, we assume that $G=c=1$, and use expressions involving the $\mathrm{BH}$ spin given by

$$
\begin{aligned}
& \Delta=r^{2}-2 M r+a^{2}, \\
& A=r^{4}+r^{2} a^{2}+2 M r a^{2}, \\
& C=1-3 r_{*}^{-1}+2 a_{*} r_{*}^{-3 / 2}, \\
& \mathcal{D}=1-2 r_{*}^{-1}+2 a_{*}^{2} r_{*}^{-2},
\end{aligned}
$$$$
\mathcal{H}=1-4 a_{*} r_{*}^{-3 / 2}+3 a_{*}^{2} r_{*}^{-2},
$$

where $a_{*}=a / M$ and $r_{*}=r / M$.

We also define

$\Omega_{\perp}^{2} \equiv \frac{M}{r^{3}} \frac{\mathcal{H}}{C}$

and a dimensionless accretion rate $\dot{m}=\dot{M} / \dot{M}_{\text {Edd }}$, where $\dot{M}_{\text {Edd }}=16 L_{\text {Edd }} / c^{2}$ is the critical accretion rate corresponding approximately to the Eddington luminosity $\left(L_{\text {Edd }}=1.25 \times\right.$ $10^{38} M / M_{\odot} \mathrm{erg} / \mathrm{s}$ ) for a disk around a non-rotating black hole.

The equations describing slim disks written in the cylindrical coordinates are:

(i) for mass conservation

$$
\dot{M}=-2 \pi \Sigma \Delta^{1 / 2} \frac{v}{\sqrt{1-v^{2}}},
$$

where $\Sigma=\int_{-h}^{+h} \rho \mathrm{d} z$ is the disk surface density, while $v$ denotes the gas velocity as measured by an observer corotating with the fluid and is related to the four-velocity $u^{r}$ by $R u^{r}=\Delta^{1 / 2} v / \sqrt{1-v^{2}}$

(ii) for radial momentum conservation

$\frac{v}{1-v^{2}} \frac{\mathrm{d} v}{\mathrm{~d} R}=\frac{\mathcal{A}}{R}-\frac{1}{\Sigma} \frac{\mathrm{d} P}{\mathrm{~d} R}$,

where

$\mathcal{A}=-\frac{M A}{R^{3} \Delta \Omega_{k}^{+} \Omega_{k}^{-}} \frac{\left(\Omega-\Omega_{k}^{+}\right)\left(\Omega-\Omega_{k}^{-}\right)}{1-\tilde{\Omega}^{2} \tilde{R}^{2}}$

and $\Omega=u^{\phi} / u^{t}$ is the angular velocity with respect to a stationary observer, $\tilde{\Omega}=\Omega-\omega$ is the angular velocity with respect to an inertial observer, $\Omega_{k}^{ \pm}= \pm M^{1 / 2} /\left(R^{3 / 2} \pm a M^{1 / 2}\right)$ are the angular frequencies of the co-rotating and counterrotating Keplerian orbits, $\tilde{R}=A /\left(R^{2} \Delta^{1 / 2}\right)$ is the radius of gyration, and $P=\int_{-h}^{+h} p \mathrm{~d} z$ is the vertically integrated total pressure;

(iii) for angular momentum conservation

$$
\frac{\dot{M}}{2 \pi}\left(\mathcal{L}-\mathcal{L}_{\text {in }}\right)=\frac{A^{1 / 2} \Delta^{1 / 2} \Gamma}{R} \alpha P,
$$

where $\mathcal{L}=u_{\phi}, \mathcal{L}_{\text {in }}$ is a constant, and $\Gamma$ is the Lorentz factor (Gammie \& Popham 1998)

$$
\Gamma^{2}=\frac{1}{1-v^{2}}+\frac{\mathcal{L}^{2} r^{2}}{A},
$$

(iv) for vertical equilibrium

$$
H^{2} \Omega_{\perp}^{2}=(2 N+3) \frac{P}{\Sigma},
$$


A. Sądowski et al.: Spinning up black holes with super-critical accretion flows

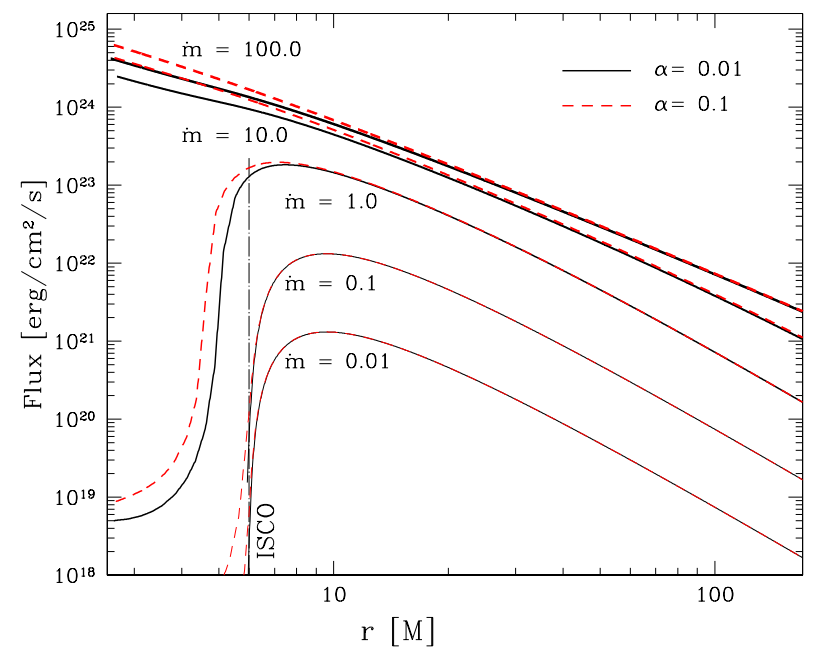

Fig. 1. Flux profiles for $M=10 M_{\odot}$ and $a_{*}=0.0$.

(v) for energy conservation

$$
\begin{aligned}
Q^{\mathrm{adv}}= & -\frac{\dot{M}}{2 \pi R^{2}}\left(\eta_{3} \frac{P}{\Sigma} \frac{\mathrm{d} \ln P}{\mathrm{~d} \ln R}-\left(1+\eta_{3}\right) \frac{P}{\Sigma} \frac{\mathrm{d} \ln \Sigma}{\mathrm{d} \ln R}\right. \\
& \left.+\eta_{3} \frac{P}{\Sigma} \frac{\mathrm{d} \ln \eta_{3}}{\mathrm{~d} \ln R}+\Omega_{\perp}^{2} \eta_{4} \frac{\mathrm{d} \ln \eta_{4}}{\mathrm{~d} \ln R}\right),
\end{aligned}
$$

where the amount of heat advected $Q^{\text {adv }}$ is

$$
Q^{\mathrm{adv}}=-\alpha P \frac{A \gamma^{2}}{R^{3}} \frac{\mathrm{d} \Omega}{\mathrm{d} r}-f_{F} \frac{64 \sigma T_{\mathrm{C}}^{4}}{3 \Sigma \kappa} .
$$

Assuming the polytropic index $N=3$, we have

$$
\begin{aligned}
& \eta_{1}=\frac{1}{T_{0}^{4}} \int_{0}^{H} T^{4} \mathrm{~d} z=128 / 315 H \\
& \eta_{2}=\frac{2}{\Sigma T_{0}} \int_{0}^{H} \rho T \mathrm{~d} z=40 / 45 \\
& \eta_{3}=\frac{1}{P}\left(\frac{1}{5 / 3-1} \frac{k}{\mu} \frac{40}{45} \Sigma T_{\mathrm{C}}+\frac{256}{315} a T_{\mathrm{C}}^{4} H\right), \\
& \eta_{4}=\frac{1}{\Sigma} \int_{0}^{H} \rho z^{2} \mathrm{~d} z=1 / 18 H^{2} .
\end{aligned}
$$

The equations given above form a two-dimensional system of ordinary differential equations with a critical (i.e., sonic) point. For each set of disk parameters, a regular solution exists for only one specific value of $\mathcal{L}_{\text {in }}$, which is an eigenvalue of the problem. The appropriate value may be found using either the relaxation or the shooting method. For details of the numerical procedures, we refer to Sạdowski (2009) and Sądowski et al. (2011).

\subsection{Disk appearance}

We now briefly describe the properties of slim disk solutions. For a more detailed discussion, we refer to e.g., Sadowski (2009), Abramowicz et al. (2010), and Bursa et al. (in prep.).

The radial profiles of the emitted flux for a non-rotating $\mathrm{BH}$ are presented in Fig. 1 . For low accretion rates $(\dot{m} \ll 1)$, they almost coincide with the Novikov \& Thorne solutions (the small departure is due to the angular momentum taken away by photons, an effect that is neglected in our slim disk scheme). When the accretion rate becomes high, advective cooling starts to play a significant role and the emission departs from that of the radiatively efficient solution. This departure is visible as early as

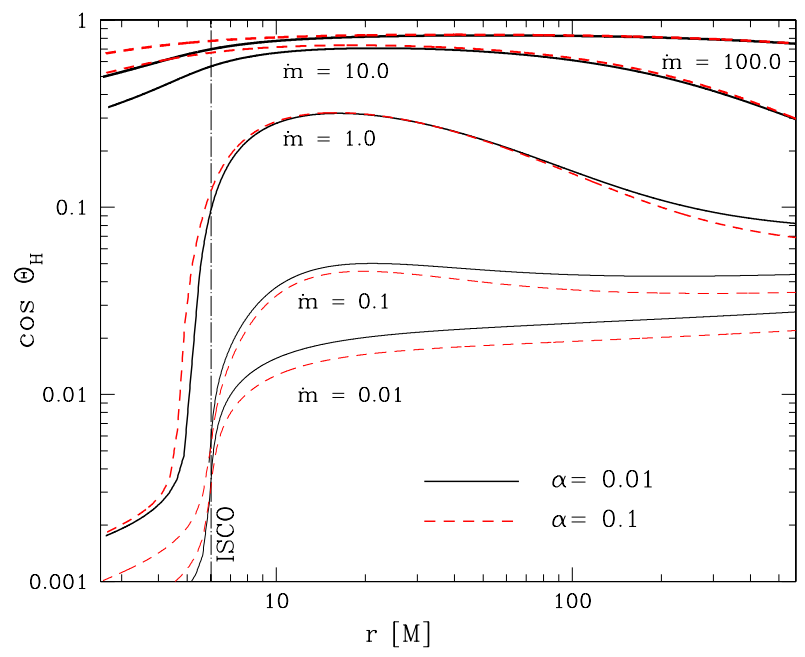

Fig. 2. Photospheric profiles for $M=10 M_{\odot}$ and $a_{*}=0.0$.

for $\dot{m}=1$ at which the emission extends significantly inside the marginally stable orbit. For super-critical accretion rates, the flux increases monotonically towards the $\mathrm{BH}$ horizon. Different colors in Fig. 1 denote solutions for different values of the viscosity parameter $\alpha$. Although the solutions are very similar, one can see that the higher the value of $\alpha$, the lower the accretion rate at which advection starts to modify the emission profile.

In Fig. 2, we plot disk thickness profiles $\left(\cos \Theta_{H}=H / r\right)$ for a range of accretion rates and two values of $\alpha$. For $\dot{m}>0.1$, the inner region of the accretion disk is puffed up by the radiation pressure and the disk surface corresponds to the location where the radiation pressure force (which is proportional to the local flux of emitted radiation) is balanced by the vertical component of the gravity force. For the Eddington accretion rate $(\dot{m} \approx 1)$, the highest $H / R$ ratio equals $\sim 0.3\left(\cos \Theta_{H} \approx 0.3\right)$, while for the highest accretion rate considered $(\dot{m}=100)$ it reaches $\sim 1.5$ $\left(\cos \Theta_{H} \approx 0.83\right)$.

In the thin disk approximation, the accreting fluid has a Keplerian angular momentum. This condition is not satisfied for advective accretion disks with significant radial pressure gradients. In Fig. 3, we present angular momentum profiles for disks with different accretion rates, $\alpha=0.01$ (left) and $\alpha=0.1$ (right panel). It is clear that the higher the accretion rate, the larger the departure from the Keplerian profile. However, the quantitative behavior depends strongly on $\alpha$. For $\alpha \lesssim 0.01$, the flow is super-Keplerian in the inner part (e.g., between $r=4.5 \mathrm{M}$ and $r=14 M$ for $\dot{m}=100)$. For larger viscosities $(\alpha \gtrsim 0.1)$ and high accretion rates, the flow is sub-Keplerian at all radii. As a result, the value of the angular momentum at the $\mathrm{BH}$ horizon $\left(\mathcal{L}_{\text {in }}\right)$ also depends strongly on $\alpha$, decreasing with increasing $\alpha$. Dependence of the flow topology on the viscosity parameter was studied in detail by Abramowicz et al. (2010).

\section{Results for bh spin evolution}

Using the slim disk solutions described in the previous section, we solve Eqs. (7) and (8) using a regular Runge-Kutta method of the 4th order. To calculate the integrals (Eqs. (11) and (12)), we use the alternative extended Simpson's rule (Press 2002) with 100 grid points in $\tilde{a}, \tilde{b}$, and radius $r$. We carried out tests to verify that this number is sufficient for convergence.

In Figs. 4 and 5, we present the BH spin evolution for $\alpha=$ 0.01 and 0.1 , respectively. The red lines show the results for different accretion rates, while the black line indicates the classical 

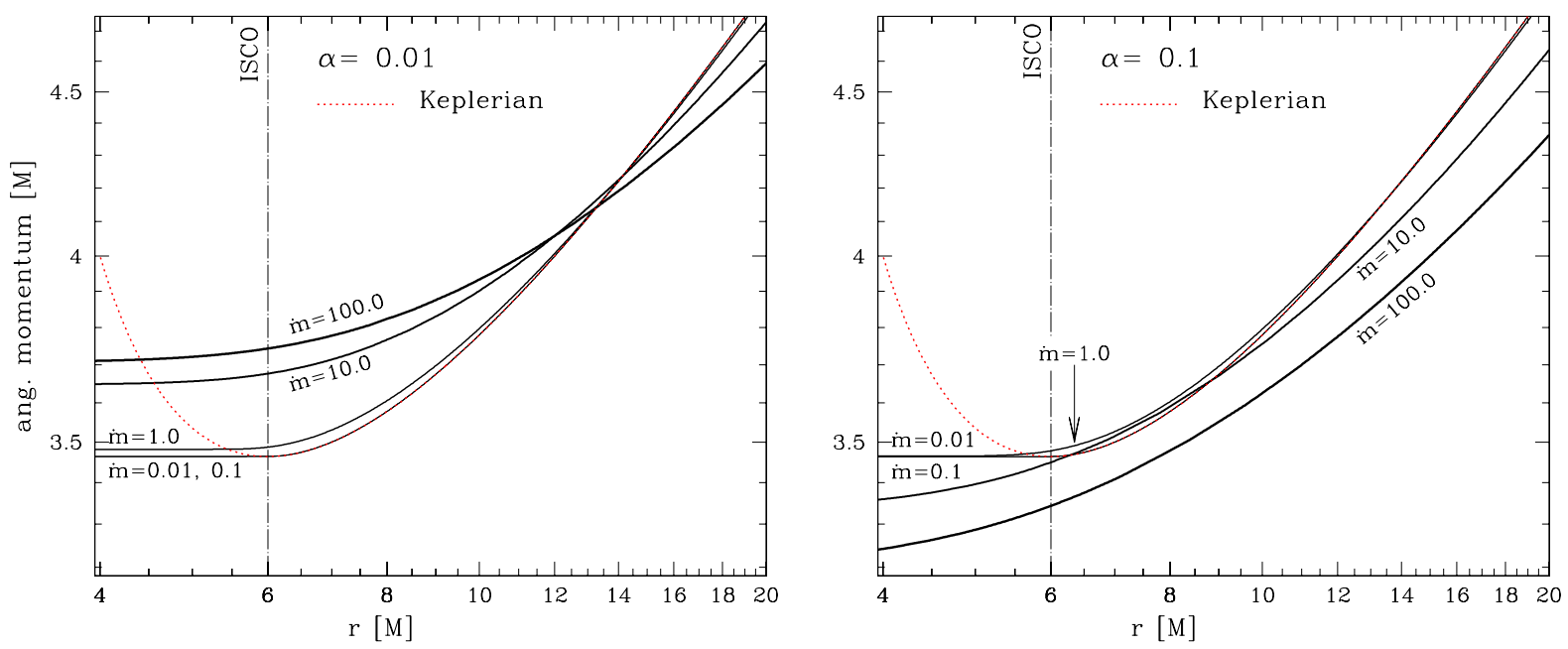

Fig. 3. Profiles of the disk angular momentum for $\alpha=0.01$ (left) and $\alpha=0.1$ (right panel) at different accretion rates in the Schwarzschild metric. The spin of the $\mathrm{BH} a_{*}=0$.

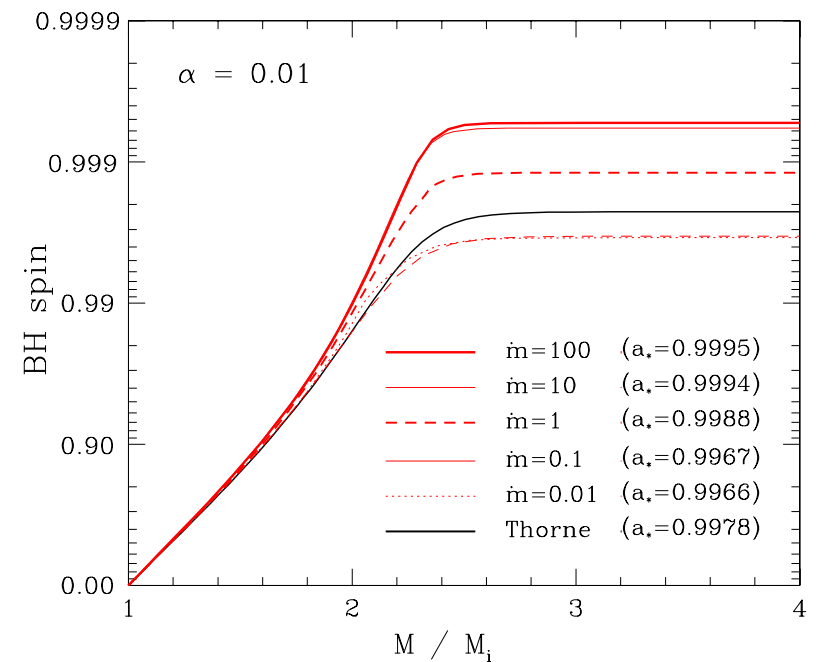

Fig. 4. Spin evolution for $\alpha=0.01$.

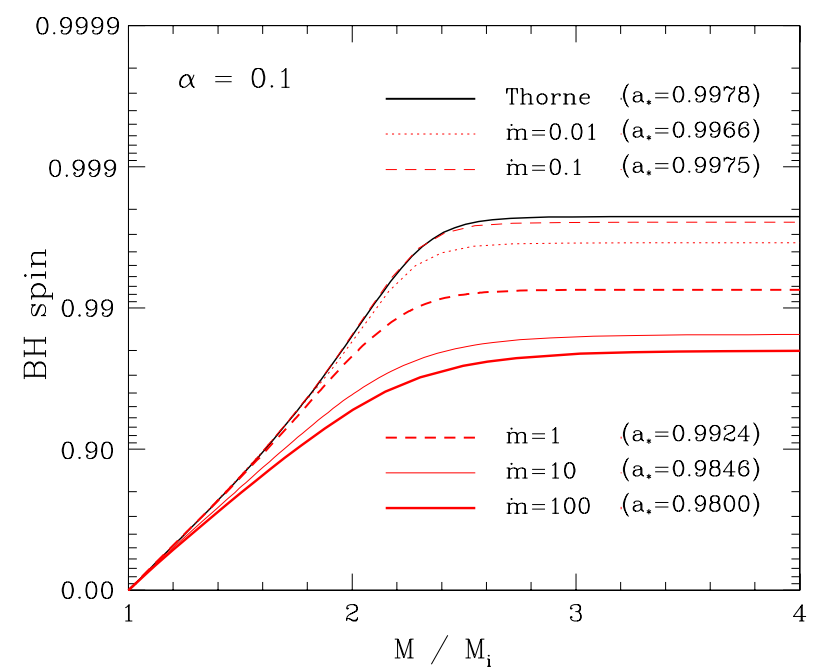

Fig. 5. Spin evolution for $\alpha=0.1$.

Thorne (1974) solution based on the Novikov \& Thorne (1973) model of thin accretion disk. Our low accretion rate limit does not perfectly agree with the black line as the slim disk model does not account for the angular momentum carried away by

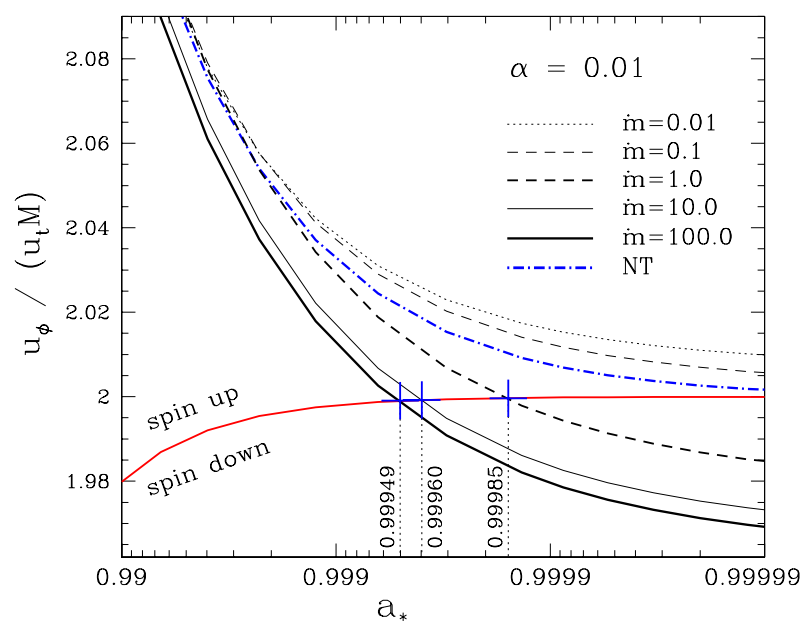

Fig. 6. The rate of spin-up or spin-down by "pure" accretion (radiation neglected) for $\alpha=0.01$. Profiles for five accretion rates are presented. Their intersections with the red line (marked with blue crosses) correspond to equilibrium states. For the two lowest accretion rates, the equilibrium state is never reached $\left(a_{*} \rightarrow 1\right)$.

radiation. As a result, the low-luminosity slim disk solutions slightly overestimate the emitted flux (by no more than a few percent) leading to stronger deceleration of the $\mathrm{BH}$ by radiation. The Thorne (1974) result is the proper limit for the lowest accretion rates. When the accretion rate is high enough (e.g., $\dot{m}>0.1$ ), the impact of the omitted angular momentum flux is overwhelmed by the modification of the disk structure introduced by advection.

To study the impact of radiation on $\mathrm{BH}$ spin evolution in detail, we calculated the rate of BH spin-up for the "pure" accretion of matter (without accounting for the impact of radiation). In that case, the $\mathrm{BH}$ spin evolution is given by (compare Eq. (7))

$\frac{\mathrm{d} a_{*}}{\mathrm{~d} \ln M}=\frac{1}{M} \frac{u_{\phi}}{u_{t}}-2 a_{*}$.

In Figs. 6 and 7, we plot with black lines the first term on the right hand side of the above equation for different accretion rates and values of $\alpha$. The red lines in these plots show the absolute value of the second term. The intersections of the black and red lines denote the equilibrium states, i.e., the limiting values 
Table 1. BH spin terminal values.

\begin{tabular}{|c|c|c|c|c|c|c|c|}
\hline \multicolumn{2}{|c|}{$\begin{array}{l}\text { Capture function: } \\
\text { model: }\end{array}$} & $\begin{array}{l}C \\
\mathbf{A}\end{array}$ & $\begin{array}{l}C \\
T\end{array}$ & $\begin{array}{l}C \\
V\end{array}$ & $\begin{array}{c}C_{1} \\
A\end{array}$ & $\begin{array}{l}C_{2} \\
A\end{array}$ & $\overline{-}$ \\
\hline \multicolumn{2}{|c|}{ thin disk } & 0.9978 & 0.9978 & 0.9978 & 0.9981 & 0.9978 & $\rightarrow 1$ \\
\hline \multirow{5}{*}{$\alpha=0.01$} & $\dot{m}=0.01$ & 0.9966 & 0.9966 & 0.9966 & 0.9975 & 0.9966 & $\rightarrow 1$ \\
\hline & $\dot{m}=0.1$ & 0.9967 & 0.9967 & 0.9967 & $\rightarrow 1$ & 0.9967 & $\rightarrow 1$ \\
\hline & $\dot{m}=1$ & 0.9988 & 0.9988 & 0.9988 & $\rightarrow 1$ & 0.9988 & 0.9998 \\
\hline & $\dot{m}=10$ & 0.9994 & 0.9994 & 0.9994 & $\rightarrow 1$ & 0.9994 & 0.9996 \\
\hline & $\dot{m}=100$ & 0.9995 & 0.9995 & 0.9995 & $\rightarrow 1$ & 0.9995 & 0.9995 \\
\hline \multirow{5}{*}{$\alpha=0.1$} & $\dot{m}=0.01$ & 0.9966 & 0.9966 & 0.9966 & 0.9975 & 0.9966 & $\rightarrow 1$ \\
\hline & $\dot{m}=0.1$ & 0.9975 & 0.9975 & 0.9975 & $\rightarrow 1$ & 0.9975 & $\rightarrow 1$ \\
\hline & $\dot{m}=1$ & 0.9924 & 0.9924 & 0.9923 & $\rightarrow 1$ & 0.9927 & 0.9948 \\
\hline & $\dot{m}=10$ & 0.9846 & 0.9846 & 0.9845 & 0.9901 & 0.9847 & 0.9951 \\
\hline & $\dot{m}=100$ & 0.9800 & 0.9800 & 0.9800 & 0.9803 & 0.9800 & 0.9801 \\
\hline
\end{tabular}

Notes. $C$ - Thorne's capture function, $C_{1}$ - all returning photons advected onto the $\mathrm{BH}, C_{2}-$ all returning photons neglected; $A-$ our fiducial model, $T$ - emission from the equatorial plane, $V$ - zero radial velocity, $N R$ - pure accretion, radiation neglected.

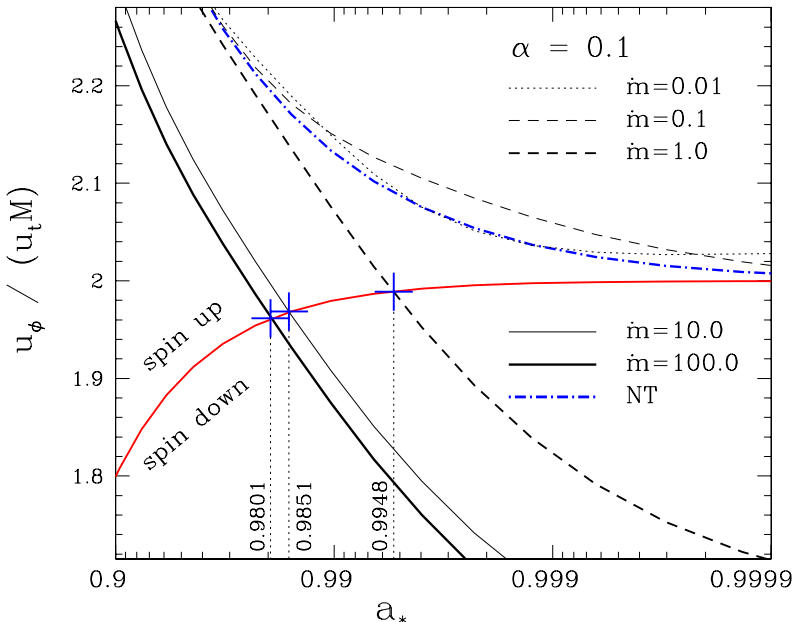

Fig. 7. Same as Fig. 6 but for $\alpha=0.1$.

of BH spin for pure accretion. These values differ significantly from the previously discussed results only at low accretion rates. In contrast, at high accretion rates radiation has little impact on the spin evolution and the value of terminal spin is mostly determined by the properties of the flow. In Fig. 8, we plot the radiation impact parameter $\xi$, defined as the ratio of the disk-driven terms on the right hand sides of Eqs. (7) and (45)

$\xi=\frac{\dot{M}_{0} u_{\phi}+\left(\frac{\mathrm{d} J}{\mathrm{~d} t}\right)_{\mathrm{rad}}}{\dot{M}_{0} u_{t}+\left(\frac{\mathrm{d} M}{\mathrm{~d} t}\right)_{\mathrm{rad}}} / \frac{u_{\phi}}{u_{t}}$.

If the captured radiation significantly decelerates the $\mathrm{BH}$ spinup, this ratio drops below unity. On the other hand, it is close to unity when the $\mathrm{BH}$ spin evolution is unaffected by the radiation. According to Fig. 8, the latter is the case for the highest accretion rates, independently of $\alpha$.

In Table 1, we list the resulting values of the terminal $\mathrm{BH}$ spin for all the models considered. The first column gives the results for our fiducial model $(A)$ including Thorne's capture function and emission from the photosphere at the appropriate radial velocity.

The second column presents results obtained assuming the same (Thorne's) capture function and profiles of emission, angular momentum, and radial velocity as in model A, but assuming the emission takes place from the equatorial plane instead of the photosphere. The resulting terminal spin values are

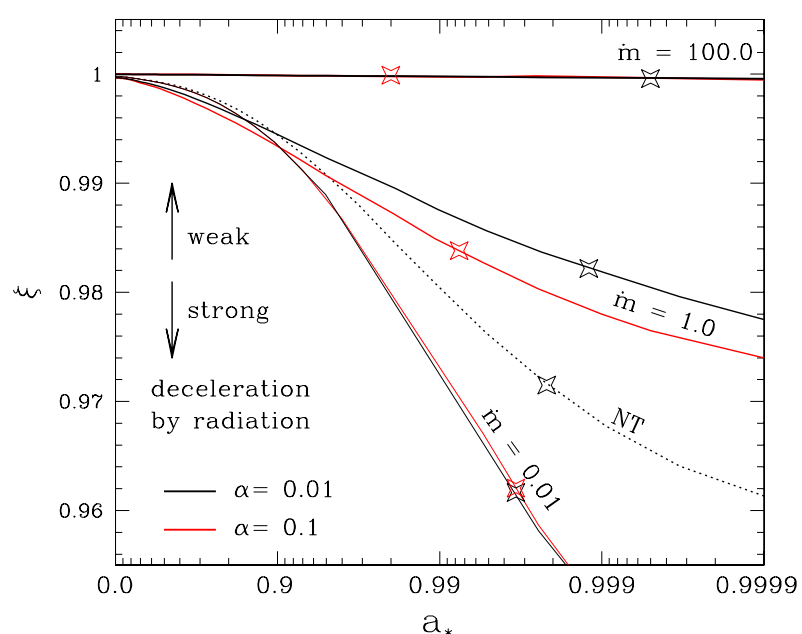

Fig. 8. Radiation impact factor $\xi$ (Eq. (46)) for different accretion rates and values of $\alpha$. The dotted line corresponds to the thin-disk induced spin evolution. For $\xi \approx 1$, spin evolution is unaffected by radiation. Stars denote the equilibrium states (compare Table 1).

equal, up to 4 decimal digits, to the values obtained with the fiducial model. This result is as expected for the lowest accretion rates, where the photosphere is located very close to the equatorial plane. For the highest accretion rates, the location of the emission has no impact on the $\mathrm{BH}$ spin-up, as the spin evolution is driven by the flow itself and the effects of radiation are negligible. However, for moderate accretion rates one could have expected significant change in the terminal spin. We find that the location of the photosphere has little impact on the resulting $\mathrm{BH}$ spin regardless of the accretion rate.

Our third model $(V)$ neglects the flow radial velocity when the radiative terms are evaluated. Similar arguments to those given in the previous paragraph apply. For the lowest accretion rates, the radial velocity is negligible and therefore should have no impact on the resulting spin. For the highest accretion rates, the spin-up process depends only on the properties of the flow. Once again, however, the impact of this assumption on moderate accretion rates is not obvious. The radial velocity turns out to be of little importance for the calculation of the terminal spin (only for $\alpha=0.1$ and moderate accretion rates the difference between models $A$ and $V$ is higher than $0.01 \%$ ).

In the fourth and fifth columns of Table 1, results for models with the same assumptions as the fiducial model, but with different capture functions are presented. The first alternative capture 
function $\left(C_{1}\right)$ assumes that the angular momentum and energy of all photons returning to the disk are added to that of the $\mathrm{BH}$. This assumption has a strong impact on the spin evolution - the terminal spin values are higher, sometimes approaching $a_{*}=1$. This may seem surprising because in the classical approach the captured photons are responsible for decelerating the spin-up. This deceleration occurs because the cross-section (with respect to the $\mathrm{BH}$ ) of photons moving "against" the frame dragging is larger than of photons following the $\mathrm{BH}$ sense of rotation. As frame dragging is involved, this effect is significant only in the vicinity of the $\mathrm{BH}$ horizon. For our model $C_{1}$, however, the probability of photons returning to the disk does not differ appreciably for co- and counter-rotating photons, as they both hit the disk surface mostly at large radii.

The other capture function $\left(C_{2}\right)$ assumes, in contrast, that all returning photons are re-emitted from the disk with their original angular momentum and energy (and never fall onto the $\mathrm{BH}$ ). This assumption cuts off the photons that would hit the $\mathrm{BH}$ in the fiducial model after crossing the disk surface, thus leading to a smaller radiative deceleration and higher values of the terminal spin parameter. However, these changes are insignificant, because most of the original photons hit the $\mathrm{BH}$ directly, along slightly curved trajectories. Only for $\alpha=0.1$ and moderate accretion rates do the terminal spin values differ in the 4th decimal digit.

Neither of the models with a modified capture functions is self-consistent. To account properly for the returning radiation, one has to modify the disk equations by introducing appropriate terms for the outgoing and incoming fluxes of angular momentum and additional radiative heating. No such model for advective, optically thick accretion disk has been constructed. The emission profile should be significantly affected (especially inside the marginally stable orbit) by the returning radiation, leading to different rates of deceleration by photons. In view of our results for models $C_{1}$ and $C_{2}$, as well as the results of $\mathrm{Li}$ et al. (2005), one may expect the final spin values for super-critical accretion flows to be slightly higher than the ones obtained in this work.

The last column of Table 1 gives terminal spin values for "pure" accretion (radiation neglected). Under these assumptions, the $\mathrm{BH}$ spin could reach $a_{*}=1$ for sub-Eddington accretion rates as there are no photons that could decelerate and stop the spin-up process. As discussed above, for the highest accretion rates the resulting $\mathrm{BH}$ spin values agree with the values obtained for the fiducial model as radiation has little impact on spin evolution in this regime.

\section{Discussion}

We have studied the spin evolution of black holes undergoing disk accretion assuming that the angular momentum and energy carried by both the flow and the emitted photons are the only factors affecting the $\mathrm{BH}$ rotation. We have generalized the original study of Thorne (1974) to high accretion rates by applying a relativistic, advective, optically thick slim accretion disk model. Assuming isotropic photon emission from the disk (no limb darkening), we have shown that:

(i) the terminal value of $\mathrm{BH}$ spin depends on the accretion rate for $\dot{m} \gtrsim 1$;

(ii) the terminal spin value is very sensitive to the assumed value of the viscosity parameter $\alpha$ - for $\alpha \lesssim 0.01$ the $\mathrm{BH}$ is spun up to $a_{*}>0.9978$ for high accretion rates, while for $\alpha \gtrsim 0.1$ to $a_{*}<0.9978$; (iii) with a low value of $\alpha$ and high accretion rates, the BH may be spun up to spins significantly higher than the canonical value $a_{*}=0.9978$ (e.g., to $a_{*}=0.9994$ for $\alpha=0.01$ and $\dot{m}=10$ ) but, under reasonable assumptions, BH cannot be spun up arbitrarily close to $a_{*}=1$;

(iv) $\mathrm{BH}$ spin evolution is hardly affected by the emitted radiation for high ( $\dot{m} \gtrsim 10$ ) accretion rates (the terminal spin value is determined by the flow properties only);

(v) for all accretion rates, neither the photosphere profile nor the profile of radial velocity significantly affects the spin evolution.

We point out that the inner edge of an accretion disk cannot be uniquely defined for super-critical accretion (Abramowicz et al. 2010), as opposed to geometrically thin disks where the inner edge is uniquely located at the marginally stable orbit $\left(R_{\mathrm{ms}}\right)$. In the thin-disk case, the BH spin evolution is determined by the flow properties at this particular radius (as there is no torque between the marginally stable orbit and $\mathrm{BH}$ horizon) and the profile of emission (terminating at $R_{\mathrm{ms}}$ ). For super-critical accretion rates, however, one cannot distinguish a particular inner edge that is relevant to studying $\mathrm{BH}$ spin evolution. On the one hand, the values of the specific energy $\left(u_{t}\right)$ and the angular momentum $\left(u_{\phi}\right)$ remain constant within the stress inner edge. On the other, the radiation is emitted outside the radiation inner edge. These inner edges do not coincide as they are related to different physical processes (Abramowicz et al. 2010).

Our study was based on a semi-analytical, hydrodynamical model of an accretion disk that makes a number of simplifying assumptions such as stationarity, no returning radiation, $\alpha$ viscosity prescription, no wind outflows, and neglects interactions of large-scale magnetic fields interactions with BHs. One has to be aware that the precise values of the terminal spin parameter are very sensitive to the flow and emission properties, as well as to the impact of magnetic fields (e.g., by means of the Blandford-Znajek process). The slim disk model only approximates the real accretion flows driven by magnetically-induced turbulence - in this respect it is no different from MHD simulations. Its applicability is limited by the adopted assumptions. The lack of magnetic fields may result in improper description of the innermost part of the flow where the disk may be be magnetically supported (Narayan et al. 2003; Igumenshchev et al. 2003; Meier 2005; Fragile \& Meier 2009). The model also does not account for the returning radiation that may affect the accretion flow. However, super-critical accretion is expected to be radiatively inefficient and therefore the impact of radiation should not be large. Despite these limitations, our study shows that Thorne's canonical value for BH spin $\left(a_{*}=0.9978\right)$ may be exceeded under certain conditions.

Acknowledgements. This work was supported in part by Polish Ministry of Science grants N203 0093/1466, N203 304035, N203 380336, and N N203 381436. J.P.L. acknowledges support from the French Space Agency CNES, MB from GAČR 205/07/0052.

\section{Appendix A: The tetrad for an observer instantaneously located at the photosphere}

Our aim is to derive the tetrad of an observer moving along the photosphere that would depend only on the quantities that are typically calculated in accretion disk models, i.e., on the radial and azimuthal velocities of gas and the location of the disk photosphere. 
The metric considered here is the Kerr geometry $g_{i k}$ in the Boyer-Lindquist coordinates $[t, \phi, r, \theta]$. The signature adopted is +--- . As in Carter's Les Houches lectures (Carter 1972), we consider two fundamental planes; the symmetry plane $\mathcal{S}_{0}=$ $[t, \phi]$ and the meridional plane $\mathcal{M}_{*}=[r, \theta]$. (Four)-vectors that belong to the plane $\mathcal{S}_{0}$, are denoted by the subscript 0 , and vectors that belong to the plane $\mathcal{M}_{*}$, will be denoted by the subscript $*$. For example, the two Killings vectors are $\eta_{0}^{i}, \xi_{0}^{i}$. We note that for any pair $X_{0}^{i}, Y_{*}^{i}$ one has,

$X_{0}^{i} Y_{*}^{k} g_{i k} \equiv\left(X_{0} Y_{*}\right)=0$.

\section{A.1. Stationary and axially symmetric photosphere}

\section{A.1.1. The photosphere}

Numerical solutions of slim accretion disks provide the location of the photosphere given by $H_{\mathrm{Ph}}(r)=r \cos \theta$. This may be substituted into $r \cos \theta-H_{\mathrm{Ph}}(r) \equiv F(r, \theta)=0$. The normal vector to the photosphere surface has the $[r, \theta]$ components

$N_{*}^{i}=\tilde{N}_{*}\left[\frac{\partial F}{\partial r}, \frac{\partial F}{\partial \theta}\right]=\tilde{N}_{*}^{\prime}\left[\frac{\mathrm{d} \theta_{*}}{\mathrm{~d} r}, 1\right]$,

where

$\frac{\mathrm{d} \theta_{*}(r)}{\mathrm{d} r}=-\frac{\partial F}{\partial r} / \frac{\partial F}{\partial \theta}$

is the derivative of the angle defining the location of the photosphere at a given radial coordinate $\left[\cos \theta_{*}(r)=H_{\mathrm{Ph}}(r) / r\right]$. Its non-zero components after normalization $\left[\left(N_{*} N_{*}\right)=-1\right]$ are

$$
\begin{aligned}
& N_{*}^{r}=\frac{\mathrm{d} \theta_{*}}{\mathrm{~d} r}\left(-g_{\theta \theta}\right)^{-1 / 2}\left[1+\frac{g_{r r}}{g_{\theta \theta}}\left(\frac{\mathrm{d} \theta_{*}}{\mathrm{~d} r}\right)^{2}\right]^{-1 / 2}, \\
& N_{*}^{\theta}=\left(-g_{\theta \theta}\right)^{-1 / 2}\left[1+\frac{g_{r r}}{g_{\theta \theta}}\left(\frac{\mathrm{d} \theta_{*}}{\mathrm{~d} r}\right)^{2}\right]^{-1 / 2} .
\end{aligned}
$$

There are two unique vectors $S_{*}$ confined to the $[r, \theta]$ plane that are orthogonal to $N_{*}$ (and therefore are tangential to the surface). From $\left(S_{*} N_{*}\right)=0$ and $\left(S_{*} S_{*}\right)=-1$, one obtains the non-zero components of one of them.

$$
\begin{aligned}
& S_{*}^{r}=\left(g_{r r}\right)^{-1}\left[-\frac{1}{g_{r r}}-\frac{1}{g_{\theta \theta}}\left(\frac{\mathrm{d} \theta_{*}}{\mathrm{~d} r}\right)^{2}\right]^{-1 / 2}, \\
& S_{*}^{\theta}=-\left(g_{\theta \theta}\right)^{-1}\left(\frac{\mathrm{d} \theta_{*}}{\mathrm{~d} r}\right)\left[-\frac{1}{g_{r r}}-\frac{1}{g_{\theta \theta}}\left(\frac{\mathrm{d} \theta_{*}}{\mathrm{~d} r}\right)^{2}\right]^{-1 / 2} .
\end{aligned}
$$

\section{A.1.2. The four-velocity of matter and the tetrad}

The four-velocity $u$ of gas moving along the photosphere may be decomposed into

$u^{i}=\tilde{A}\left(u_{0}^{i}+v S_{*}^{i}\right)$,

where

$u_{0}^{i}=\tilde{A}_{0}\left(\eta^{i}+\Omega \xi^{i}\right)$

is the four-velocity of an observer with azimuthal motion only. The normalization constant $\tilde{A}_{0}$ comes from $\left(u_{0} u_{0}\right)=1$ and equals

$\tilde{A}_{0}=\left[g_{t t}+\Omega g_{\phi \phi}(\Omega-2 \omega)\right]^{-1 / 2}$.
It is useful to construct a spacelike vector $\left(\kappa_{0}\right)$ confined to the $[t, \phi]$ plane, that is perpendicular to both $u$ and $u_{0}$. From $(\kappa \kappa)=-1$ and, e.g., $\left(\kappa u_{0}\right)=0$, we have

$\kappa_{0}^{i}=\frac{\left(l \eta^{i}+\xi^{i}\right)}{\left[-g_{\phi \phi}(1-\Omega l)(1-\omega l)\right]^{1 / 2}}$,

where $l=u_{\phi} / u_{t}$ is the specific angular momentum. We note that the set of vectors $\left[u_{0}^{i}, N_{*}^{i}, \kappa_{0}^{i}, S_{*}^{i}\right]$ already forms the desired tetrad that is valid for the pure rotation $\left(u^{r}=0\right)$ case.

The normalization condition $(u u)=1$ gives

$\tilde{A}=\left[g_{t t}+\Omega g_{\phi \phi}(\Omega-2 \omega)-v^{2}\right]^{-1 / 2}$,

where $v$ is related to the radial component of the gas fourvelocity $u^{r}$ by

$v^{2}=\frac{\left(u^{r} / S_{*}^{r}\right)^{2}\left[g_{t t}+\Omega g_{\phi \phi}(\Omega-2 \omega)\right]}{1+\left(u^{r} / S_{*}^{r}\right)^{2}}$.

The vectors we have just calculated $\left(u, \kappa_{0}\right)$ are both orthogonal to $N_{*}$ since $\left(N_{*} S_{*}\right)=0$. To complete the tetrad, we need one more spacelike vector $(S)$ that is orthogonal to these three. We decompose this into

$S^{i}=\alpha u^{i}+\beta \kappa_{0}^{i}+\gamma N_{*}^{i}+\delta S_{*}^{i}$.

The orthogonality conditions $\left(\kappa_{0} S\right)=0$ and $\left(N_{*} S\right)=0$ immediately implies that $\gamma=\beta=0$. The only non-trivial condition is that $(u S)=0$. Together with $(S S)=-1$, it implies that

$S^{i}=\left(1+\tilde{A}^{2} v^{2}\right)^{-1 / 2}\left(\tilde{A} v u^{i}+S_{*}^{i}\right)$.

The vectors $u^{i}, N_{*}^{i}, \kappa_{0}^{i}$, and $S^{i}$ form an orthonormal tetrad in the Kerr spacetime

$e_{(A)}^{i}=\left[u^{i}, N_{*}^{i}, \kappa_{0}^{i}, S^{i}\right]$.

This tetrad is known directly from the slim disk solutions, as it depends on the calculated quantities $\left(u^{r}, \Omega, l\right.$ and $\left.\theta_{*}(r)\right)$ only. Any spacetime vector $X^{i}$ could be uniquely decomposed into this tetrad with $X_{(A)}=X_{i} e_{(A)}^{i}$.

\section{A.2. The general case}

We here assume nothing about the four-velocity of matter $u^{i}$ and the location of photosphere. Both may be non-stationary and non-axially symmetric. Following the same framework as in Sect. A.1, we describe how to obtain the tetrad of an observer instantaneously located at the photosphere that depends only on the quantities calculated by accretion disk models.

\section{A.2.1. The four velocity}

As in Sect. A.1.2, we may always uniquely decompose $u^{i}$, a general timelike unit vector, into

$u^{i}=\tilde{A}\left(u_{0}^{i}+v S_{*}^{i}\right)$

where $u_{0}^{i}$ is a timelike unit vector, and $S_{*}^{i}$ is a spacelike unit vector. Equation (A.14) uniquely defines the two vectors $u_{0}^{i}, S_{*}^{i}$ and the two scalars $\tilde{A}, v$. The vectors and scalars

$\left\{\tilde{A}, v, u_{0}^{i}, S_{*}^{i}\right\}$, 
can be calculated from known quantities given by slim disk model solutions.

The four-velocity (A.14) also defines the instantaneous 3space of the comoving observer with the metric $\gamma_{i k}$ and the projection tensor $h_{k}^{i}$

$$
\begin{aligned}
\gamma_{i k} & =g_{i k}-u_{i} u_{k}, \\
h_{k}^{i} & =\delta_{k}^{i}-u^{i} u_{k} .
\end{aligned}
$$

We define the two unit vectors $\kappa_{0}^{i}$ and $N_{*}^{i}$ by the unique condition

$\left(\kappa_{0} u_{0}\right)=0, \quad\left(S_{*} N_{*}\right)=0$.

As before, the four vectors

$e_{(A)}^{i}=\left[u_{0}^{i}, N_{*}^{i}, \kappa_{0}^{i}, S_{*}^{i}\right]$,

form an orthonormal tetrad of an observer with the fourvelocity $u_{0}^{i}$ can calculated from the solutions of the slim-disk equations.

\section{A.2.2. The photosphere}

In the most general case of a non-stationary and non-axially symmetric photosphere, the location of the photosphere may be described by the condition

$F(t, \phi, r, \theta)=0$.

The vector $\tilde{N}$ normal to the photosphere has the components

$\tilde{N}^{i}=\left[\frac{\partial F}{\partial t}, \frac{\partial F}{\partial \phi}, \frac{\partial F}{\partial r}, \frac{\partial F}{\partial \theta}\right]$

which may be calculated from slim disk solutions.

We project $\tilde{N}$ into the instantaneous 3 -space of the comoving observer (A.17) and normalize to a unit vector after the projection to obtain

$N^{i}=\frac{\hat{N}^{i}}{|(\hat{N} \hat{N})|^{1 / 2}}, \quad \hat{N}^{i}=\tilde{N}^{k} h_{k}^{i}$.

In terms of the tetrad in Eq. (A.19), a vector $N^{i}$ constructed in this way has the decomposition

$N^{i}=\tilde{N}\left[\alpha\left(u_{0}^{i}\right)+1\left(N_{*}^{i}\right)+\gamma\left(\kappa_{0}^{i}\right)+\delta\left(S_{*}^{i}\right)\right]$.

The components $\tilde{N}, \alpha, \gamma, \delta$ are known.

\section{A.2.3. The tetrad}

We now decompose the four vectors, the first two of which we have derived, the next two guessed (but the guess should be obvious):

$$
\begin{aligned}
u^{i} & =\tilde{A}\left[1\left(u_{0}^{i}\right)+0\left(N_{*}^{i}\right)+0\left(\kappa_{0}^{i}\right)+V\left(S_{*}^{i}\right)\right], \\
N^{i} & =\tilde{N}\left[\alpha\left(u_{0}^{i}\right)+1\left(N_{*}^{i}\right)+\gamma\left(\kappa_{0}^{i}\right)+\delta\left(S_{*}^{i}\right)\right], \\
\kappa^{i} & =\tilde{\kappa}\left[0\left(u_{0}^{i}\right)+b\left(N_{*}^{i}\right)+1\left(\kappa_{0}^{i}\right)+0\left(S_{*}^{i}\right)\right], \\
S^{i} & =\tilde{S}\left[A\left(u_{0}^{i}\right)+B\left(N_{*}^{i}\right)+C\left(\kappa_{0}^{i}\right)+1\left(S_{*}^{i}\right)\right] .
\end{aligned}
$$

The four unknown components, $b, A, B, C$ one calculates from the following four non-trivial orthogonality conditions $((u \kappa) \equiv 0$ by construction, $c f .(A .24)$ and (A.26))

$(u S)=0, \quad(N S)=0, \quad(S \kappa)=0, \quad(N \kappa)=0$, and the two unknown factors $\tilde{\kappa}$ and $\tilde{S}$ from the following two normalization conditions

$(\kappa \kappa)=-1, \quad(S S)=-1$.

The conditions (A.28) and (A.29) are given by linear equations.

Equations (A.24)-(A.29) define the tetrad $e_{(A)}^{i}$ of an observer comoving with matter, and instantaneously located at the photosphere:

$e_{(A)}^{i}=\left[u^{i}, N^{i}, \kappa^{i}, S^{i}\right]$.

Both the matter and the photosphere move in a general manner. The zenithal direction in the local observer's sky is given by $N^{i}$.

\section{Appendix B: Integration over the world-tube of the photosphere}

For stationary and axially symmetric models, we define:

$N^{i}=N_{*}^{i}=$ unit vector orthogonal to the photosphere, which is in the $[r, \theta]$ plane;

$S_{*}^{i}=$ unit vector orthogonal to $N^{i}$, which is in the $[r, \theta]$ plane;

$u^{i}=$ four-velocity of matter, which is in the $[t, \phi, r, \theta]$ spacetime;

$\kappa^{i}=\kappa_{0}^{i}=$ unit vector orthogonal to $U^{i}$, which is in the $[t, \phi]$ plane;

$S^{i}=$ unit vector orthogonal to $U^{i}, N^{i}$ and $\kappa^{i}$, which is in the $[t, \phi, r, \theta]$ space-time;

$e_{(A)}^{i}=\left[u^{i}, N^{i}, \kappa^{i}, S^{i}\right]=$ the tetrad comoving with an observer located in the photosphere.

The integration of a vector $(\ldots)_{i}$ over the $3 \mathrm{D}$ hypersurface $\mathcal{H}$ orthogonal to $N^{i}$ (i.e. the 3D world-tube of the photosphere) may be symbolically written as

$\int_{\mathcal{H}}(\ldots)_{i} N^{i} \mathrm{~d} S$

where $\mathrm{d} S$ is the "volume element" in $\mathcal{H}$.

Obviously, the hypersurface $\mathcal{H}$ is spanned by the three vectors $\left[u^{i}, \kappa^{i}, S^{i}\right]_{N}$. Each of them is a linear combination of $\left[\eta^{i}, \xi^{i}, S_{*}^{i}\right]_{N}$, and each of the three vectors from $\left[\eta^{i}, \xi^{i}, S_{*}^{i}\right]_{N}$ is orthogonal to $N^{i}$.

Therefore, one may say that the hypersurface $\mathcal{H}$ is spanned by $\left[\eta^{i}, \xi^{i}, S_{*}^{i}\right]_{N}$. It is convenient to write

$\mathrm{d} S=\mathrm{d} A \mathrm{~d} R, \quad \mathrm{~d} R=\mathrm{d} r \sqrt{g_{r r}+g_{\theta \theta}\left(\frac{\mathrm{d} \theta_{*}}{\mathrm{~d} r}\right)^{2}}$,

where $\mathrm{d} R$ is the line element along the vector $S_{*}^{i}$, i.e. along the photosphere in the $[r, \theta]$ plane, with $\theta=\theta_{*}(r)$ defining the location of the photosphere, and $\mathrm{d} A$ being the surface element on the $[t, \phi]$ plane.

To calculate $\mathrm{d} A$ we imagine an infinitesimal parallelogram with sides that are located along the $t=$ const. and $\phi=$ const. lines. The proper lengths of the sides are $\mathrm{d} u=\left|g_{t t}\right|^{1 / 2} \mathrm{~d} t$ and $\mathrm{d} v=$ $\left|g_{\phi \phi}\right|^{1 / 2} \mathrm{~d} \phi$, respectively, and therefore $\mathrm{d} A$, which is just the area of the parallelogram, is given by

$\mathrm{d} A=\mathrm{d} u \mathrm{~d} v \sin \alpha=\mathrm{d} t \mathrm{~d} \phi\left|g_{t t}\right|^{1 / 2}\left|g_{\phi \phi}\right|^{1 / 2} \sin \alpha$,

where $\alpha$ is the angle between the two sides. Obviously, the cosine of this angle is given by the scalar product of the two unit 
vectors $n_{i}$ and $x_{i}$ pointing in the $[t, \phi]$ plane into the $t$ and $\phi$ directions respectively. These vectors are given by (note that $n_{i}=$ ZAMO)

$n_{i}=\frac{\left(\nabla_{i} t\right)}{\left|g^{j k}\left(\nabla_{j} t\right)\left(\nabla_{k} t\right)\right|^{1 / 2}}, \quad x_{i}=\frac{\left(\nabla_{i} \phi\right)}{\left|g^{j k}\left(\nabla_{j} \phi\right)\left(\nabla_{k} \phi\right)\right|^{1 / 2}}$.

Because $\left(\nabla_{i} t\right)=\delta_{i}^{t}$ and $\left(\nabla_{i} \phi\right)=\delta_{i}^{\phi}$, one may write

$n_{i}=\frac{\delta_{i}^{t}}{\left|g^{t t}\right|^{1 / 2}}, \quad x_{i}=\frac{\delta_{i}^{\phi}}{\left|g^{\phi \phi}\right|^{1 / 2}}$.

Therefore,

$\cos \alpha=n_{i} x_{k} g^{i k}=\frac{g^{t \phi}}{\left|g^{t t}\right|^{1 / 2}\left|g^{\phi \phi}\right|^{1 / 2}}=-\frac{g_{t \phi}}{\left|g_{t t}\right|^{1 / 2}\left|g_{\phi \phi}\right|^{1 / 2}}$,

and

$\sin \alpha=\frac{\left(g_{t \phi}^{2}-g_{t t} g_{\phi \phi}\right)^{1 / 2}}{\left|g_{t t}\right|^{1 / 2}\left|g_{\phi \phi}\right|^{1 / 2}}$.

Inserting this into the formula for $\mathrm{d} A$, we get $\mathrm{d} A=\mathrm{d} t \mathrm{~d} \phi\left(g_{t \phi}^{2}-\right.$ $\left.g_{t t} g_{\phi \phi}\right)^{1 / 2}$. The final formula for $\mathrm{d} S$ is,

$\mathrm{d} S=\mathrm{d} t \mathrm{~d} \phi \mathrm{d} r\left(g_{t \phi}^{2}-g_{t t} g_{\phi \phi}\right)^{1 / 2} \sqrt{g_{r r}+g_{\theta \theta}\left(\frac{\mathrm{d} \theta_{*}}{\mathrm{~d} r}\right)^{2}}$.

\section{References}

Abramowicz, M. A., \& Lasota, J. P. 1980, Acta Astron., 30, 35 Abramowicz, M. A., Chen, X.-M., Granath, M., \& Lasota, J.-P. 1996, ApJ, 471, 762

Abramowicz, M. A., Jaroszyński, M., Kato, S., et al. 2010, A\&A, 521, A15 Bañados, M., Silk, J., \& West, S. M. 2009, Phys. Rev. Lett., 103, 111102
Bardeen, J. M. 1970, Nature, 226, 64

Bardeen, J. M., Press, W. H., \& Teukolsky, S. A. 1972, ApJ, 178, 347

Bardeen, J. M., Carter, B., \& Hawking, S. W. 1973, Comm. Math. Phys., 31, 161 Belczynski, K., Taam, R. E., Rantsiou, E., \& van der Sluys, M. 2008, ApJ, 682, 474

Berti, E., \& Volonteri, M. 2008, ApJ, 684, 822

Bursa, M. 2006, Ph.D. Thesis

Carter B. 1973, in Black Holes (Les Astres Occlus), ed. C. DeWitt, \& B. S. DeWitt (Gordon and Breach Science Publishers), 57

Fanidakis, N., Baugh, C. M., Benson, A. J., et al. 2011, MNRAS, 410, 53

Fragile, P. C., \& Meier, D. L. 2009, ApJ, 693, 771

Gammie, C. F., \& Popham, R. 1998, ApJ, 498, 313

Gammie, C. F., Shapiro, S. L., \& McKinney, J. C. 2004, ApJ, 602, 312

Ghosh, P., \& Abramowicz, M. A. 1997, MNRAS, 292, 887

Igumenshchev, I. V., Narayan, R., \& Abramowicz, M. A. 2003, ApJ, 592, 1042

Kato, S., Fukue, J., \& Mineshige, S. 2008, Black-Hole Accretion Disks Towards a New Paradigm, ed. S. F. J. M. S. Kato

King, A. R., Pringle, J. E., \& Hofmann, J. A. 2008, MNRAS, 385, 1621

Kozłowski, M., Jaroszyński, M., \& Abramowicz, M. A. 1978, A\&A, 63, 209

Lasota, J. P. 1994, in Theory of Accretion Disks - 2, ed. W. J. Duschl, J. Frank, F. Meyer, E. Meyer-Hofmeister, \& W. M. Tscharnuter, NATO ASIC Proc., 417: 341

Li, L.-X., Zimmerman, E. R., Narayan, R., \& McClintock, J. E. 2005, ApJS, 157, 335

Livio, M., Ogilvie, G. I., \& Pringle, J. E. 1999, ApJ, 512, 100

McKinney, J. C., \& Narayan, R. 2007, MNRAS, 375, 513

Meier, D. L. 2005, Ap\&SS, 300, 55

Misner, C. W., Thorne, K. S., \& Wheeler, J. A. 1973 (San Francisco: W.H. Freeman and Co.)

Moderski, R., Sikora, M., \& Lasota, J.-P. 1998, MNRAS, 301, 142

Narayan, R., Igumenshchev, I. V., \& Abramowicz, M. A. 2003, PASJ, 55, L69

Novikov, I. D., \& Thorne, K. S. 1973, in Black Holes (Les Astres Occlus), ed. C. DeWitt, \& B. S. DeWitt (Gordon and Breach Science Publishers), 343 Ohsuga, K. 2007, ApJ, 659, 205

Popham, R., \& Gammie, C. F. 1998, ApJ, 504, 419

Press, W. H. 2002, Numerical recipes in $\mathrm{C}++$ : the art of scientific computing by William H. Press

Shakura, N. I., \& Sunyaev, R. A. 1973, A\&A, 24, 337

Sadowski, A. 2009, ApJS, 183, 171

Sądowski, A., Abramowicz, M., Bursa, M., et al. 2011, A\&A, 527, A17

Thorne, K. S. 1974, ApJ, 191, 507

Volonteri, M., Madau, P., Quataert, E., \& Rees, M. J. 2005, ApJ, 620, 69

Volonteri, M., Sikora, M., \& Lasota, J.-P. 2007, ApJ, 667, 704

Wald, R. W. 1984 (University of Chicago Press) 